This item was submitted to Loughborough's Research Repository by the author.

Items in Figshare are protected by copyright, with all rights reserved, unless otherwise indicated.

\title{
On a class of third-order nonlocal Hamiltonian operators
}

PLEASE CITE THE PUBLISHED VERSION

https://doi.org/10.1016/j.geomphys.2018.10.018

PUBLISHER

(c) Elsevier

VERSION

AM (Accepted Manuscript)

PUBLISHER STATEMENT

This paper was accepted for publication in the journal Journal of Geometry and Physics and the definitive published version is available at https://doi.org/10.1016/j.geomphys.2018.10.018.

\section{LICENCE}

CC BY-NC-ND 4.0

\section{REPOSITORY RECORD}

Casati, M., Evgeny Ferapontov, Maxim V. Pavlov, and R.F. Vitolo. 2019. "On a Class of Third-order Nonlocal Hamiltonian Operators". figshare. https://hdl.handle.net/2134/35764. 


\title{
On a class of third-order nonlocal Hamiltonian operators
}

\author{
M. Casati ${ }^{1}$, E.V. Ferapontov ${ }^{1}$, M.V. Pavlov ${ }^{2}$, R.F. Vitolo ${ }^{3}$ \\ ${ }^{1}$ Department of Mathematical Sciences, \\ Loughborough University, \\ Loughborough, Leicestershire, LE11 3TU, UK \\ e.v.ferapontov@lboro.ac.uk \\ 2 Lebedev Physical Institute of Russian Academy of Sciences, \\ Leninskij Prospekt 53, 119991 Moscow, Russia \\ $\mathrm{m} \cdot \mathrm{v} \cdot$ pavlov@lboro.ac.uk \\ ${ }^{3}$ Department of Mathematics and Physics "E. De Giorgi", \\ University of Salento, Lecce, Italy \\ and INFN, Section of Lecce \\ raffaele.vitolo@unisalento.it
}

\begin{abstract}
Based on the theory of Poisson vertex algebras we calculate skew-symmetry conditions and Jacobi identities for a class of third-order nonlocal operators of differential-geometric type. Hamiltonian operators within this class are defined by a Monge metric and a skew-symmetric two-form satisfying a number of differentialgeometric constraints. Complete classification results in the 2-component and 3component cases are obtained.
\end{abstract}

MSC: 37K05, 37K10, 37K20, 37K25.

Keywords: Nonlocal Hamiltonian Operator, Monge Metric, Dirac Reduction, Poisson Vertex Algebra. 


\section{Contents}

1 Introduction and summary of the main results $\quad 2$

2 Example $\quad 4$

3 Classification results 4

3.1 2-component case . . . . . . . . . . . . . . . . 5 5

3.23 -component case . . . . . . . . . . . . . . . . 6

4 Nonlocal operators via Dirac reduction $\quad 10$

5 Skew-symmetry conditions and Jacobi identities: proof of Theorem 112

5.1 The $\lambda$ bracket . . . . . . . . . . . . . . . . . 14

5.2 The skew-symmetry condition . . . . . . . . . . . . . . . 14

5.3 The PVA-Jacobi identity . . . . . . . . . . . . . . . . 15

6 Concluding Remarks $\quad 17$

7 Acknowledgements $\quad 17$

\section{Introduction and summary of the main results}

Third-order Hamiltonian operators of differential-geometric type were introduced in [6] and thoroughly investigated in $[23,25,24,1,5]$. In the so-called 'flat coordinates' $u=$ $\left\{u^{1}, \ldots, u^{n}\right\}$ these operators take the form

$$
A=\partial_{x}\left(g^{i j} \partial_{x}+c_{k}^{i j} u_{x}^{k}\right) \partial_{x}
$$

where the coefficients $g^{i j}(u)$ and $c_{k}^{i j}(u)$ satisfy a system of differential constraints coming from the skew-symmetry conditions and the Jacobi identities. Here $i, j, k \in\{1, \ldots, n\}$ where $n$ is the number of components. Hamiltonian operators of type (1) arise in the theory of equations of associativity of 2-dimensional topological field theory (WDVV equations [7]), see [9, 19, 16, 17, 20]. Projective-geometric aspects of operators (1) were studied in [11, 12] based on their correspondence to Monge metrics and quadratic line complexes. This has lead to complete classification results for the number of components $n \leq 4$. The general theory of Hamiltonian systems of conservation laws associated with operators (1) was developed in [13].

In this paper we investigate a nonlocal generalisation of ansatz (1),

$$
A=\partial_{x}\left(g^{i j} \partial_{x}+c_{k}^{i j} u_{x}^{k}+w_{k}^{i} u_{x}^{k} \partial_{x}^{-1} w_{l}^{j} u_{x}^{l}\right) \partial_{x}
$$

in what follows we will always assume the non-degeneracy condition $\operatorname{det} g \neq 0$. Operator of type (2) appeared in the context of the Wadati-Konno-Ishikawa (WKI) soliton hierarchy [26, 18], see Section 2. Although operator (2) looks analogous to first-order nonlocal Hamiltonian operators introduced in [8], the underlying geometry is quite different. 
Theorem 1. Operator (2) is Hamiltonian if and only if the following conditions are satisfied:

$$
\begin{gathered}
g^{i j}=g^{j i}, \\
g_{, k}^{i j}=c_{k}^{i j}+c_{k}^{j i}, \\
c_{s}^{i j} g^{s k}+c_{s}^{k j} g^{s i}=0, \\
c_{s}^{i j} g^{s k}+c_{s}^{j k} g^{s i}+c_{s}^{k i} g^{s j}=0, \\
g^{i s} w_{s}^{k}+g^{k s} w_{s}^{i}=0, \\
g^{k s} w_{s, l}^{j}+g_{, l}^{j s} w_{s}^{k}-c_{s}^{j k} w_{l}^{s}+c_{s}^{k j} w_{l}^{s}=0, \\
g^{k s} c_{s, l}^{i j}+c_{s}^{k j} g_{, l}^{s i}+c_{s}^{k i} c_{l}^{s j}-c_{s}^{i k} c_{l}^{s j}+g^{k s} w_{s}^{i} w_{l}^{j}=0 .
\end{gathered}
$$

Our proof of Theorem 1 utilises the theory of Poisson vertex algebras, see Section 5 . Let us introduce an affine connection $\nabla$ with Christoffel's symbols $\Gamma_{j k}^{i}=c_{j k}^{i}=g_{j s} c_{k}^{s i}, c_{i j k}=$ $g_{i s} c_{j k}^{s}$ where $g_{i j}$ is the inverse matrix to $g^{i j}$. Note that Christoffel's symbols are skewsymmetric in low indices. Introducing the skew-symmetric 2-form $w_{i j}=g_{i s} w_{j}^{s}$ we can rewrite conditions (3) in the equivalent form with low indices,

$$
\begin{gathered}
\nabla g=0, \\
c_{i j k}+c_{i k j}=0, \\
g_{i j, k}+g_{j k, i}+g_{k i, j}=0, \\
w_{i j}+w_{j i}=0, \\
w_{i j, l}-c_{i j}^{s} w_{s l}=0, \\
c_{n m l, k}+c_{m l}^{s} c_{s n k}+w_{m l} w_{n k}=0 .
\end{gathered}
$$

The last relation implies

$$
R_{i j k l}=w_{i l} w_{j k}-w_{i k} w_{j l}
$$

where $R_{i j k l}=g_{i s} R_{j k l}^{s}$ is the curvature tensor of the connection $\nabla$. Note that a metric $g$ satisfying the equations $g_{i j, k}+g_{j k, i}+g_{k i, j}=0$ is the Monge metric of a quadratic line complex in $\mathbb{P}^{n}[11,12]$. The existing classification of such metrics in dimensions 2 and 3 leads to a complete list of 2-component (Theorem 2 of Section 3.1) and 3-component (Theorem 3 of Section 3.2) operators (2). An important invariant of a Monge metric is its singular variety defined by the equation det $g=0$. The singular variety is an algebraic hypersurface of degree $2 n-2$ [4]. For local operators (1) the singular variety is known to be a double hypersurface of degree $n-1$ [11]. This is no longer the case for nonlocal operators (2): the corresponding singular varieties are generally irreducible.

In Theorem 4 of Section 4 we demonstrate that $n$-component nonlocal operators (2) arise as Dirac reductions of $(n+1)$-component local operators $(1)$ to hyperplanes in the flat coordinates.

Remark. The first three conditions (3) imply that the coefficients $c_{k}^{i j}$ can be expressed in terms of the metric by the formula [11]

$$
c_{k}^{i j}=\frac{1}{3} g^{q i} g^{p j}\left(g_{p k, q}-g_{p q, k}\right) .
$$

Setting $k=j, l=i$ in the relation $R_{i j k l}=w_{i l} w_{j k}-w_{i k} w_{j l}$ we obtain $R_{i j j i}=w_{i j}^{2}$, which determines $w_{i j}=\sqrt{R_{i j j i}}$ uniquely up to a sign (which can be fixed in a consistent way from the remaining relations up to the overall sign, $w \rightarrow-w)$. Thus, to specify Hamiltonian operator (2) it is sufficient to specify the corresponding Monge metric $g_{i j}$. 


\section{Example}

The second flow of the so(3) version of the WKI hierarchy [26] has the form

$$
\left(\begin{array}{c}
p \\
q
\end{array}\right)_{t}=\left(\frac{\frac{p_{x}}{\left(p^{2}+q^{2}+1\right)^{3 / 2}}}{\left(p^{2}+q^{2}+1\right)^{3 / 2}}\right)_{x x} .
$$

It was demonstrated in [18] that this system possesses a bi-Hamiltonian representation

$$
\left(\begin{array}{c}
p \\
q
\end{array}\right)_{t}=A\left(\begin{array}{c}
\delta H / \delta p \\
\delta H / \delta q
\end{array}\right)=B\left(\begin{array}{c}
\delta G / \delta p \\
\delta G / \delta q
\end{array}\right)
$$

with the Hamiltonians

$$
H=\int \sqrt{p^{2}+q^{2}+1} d x, \quad G=\int \frac{q p_{x}-p q_{x}}{\sqrt{p^{2}+q^{2}+1}\left(\sqrt{p^{2}+q^{2}+1}+1\right)} d x,
$$

and the Hamiltonian operators

$$
A=\partial_{x}^{2}\left(\begin{array}{cc}
\partial_{x}^{-1}-\tilde{q} \partial_{x}^{-1} \tilde{q} & \tilde{q} \partial_{x}^{-1} \tilde{p} \\
\tilde{p} \partial_{x}^{-1} \tilde{q} & \partial_{x}^{-1}-\tilde{p} \partial_{x}^{-1} \tilde{p}
\end{array}\right) \partial_{x}^{2}, \quad B=\left(\begin{array}{cc}
0 & 1 \\
-1 & 0
\end{array}\right) \partial_{x}^{2},
$$

where we use the notation

$$
\tilde{p}=\frac{p}{\sqrt{p^{2}+q^{2}+1}}, \quad \tilde{q}=\frac{q}{\sqrt{p^{2}+q^{2}+1}} .
$$

The operator $A$ can be rewritten in form (2),

$$
A=\partial_{x} \circ A_{1} \circ \partial_{x}
$$

where

$$
A_{1}=\left(\begin{array}{cc}
1-\tilde{q}^{2} & \tilde{q} \tilde{p} \\
\tilde{p} \tilde{q} & 1-\tilde{p}^{2}
\end{array}\right) \partial_{x}+\left(\begin{array}{cc}
-\tilde{q} \tilde{q}_{x} & \tilde{p} \tilde{q}_{x} \\
\tilde{q} \tilde{p}_{x} & -\tilde{p} \tilde{p}_{x}
\end{array}\right)+\left(\begin{array}{cc}
\tilde{q}_{x} \partial_{x}^{-1} \tilde{q}_{x} & -\tilde{q}_{x} \partial_{x}^{-1} \tilde{p}_{x} \\
-\tilde{p}_{x} \partial_{x}^{-1} \tilde{q}_{x} & \tilde{p}_{x} \partial_{x}^{-1} \tilde{p}_{x}
\end{array}\right)
$$

For the corresponding Monge metric $g_{i j}$ and the skew-symmetric 2-form $w_{i j}$ we obtain

$$
g_{i j}=\left(\begin{array}{cc}
q^{2}+1 & -p q \\
-p q & p^{2}+1
\end{array}\right), \quad w_{i j}=\frac{1}{\sqrt{p^{2}+q^{2}+1}}\left(\begin{array}{cc}
0 & 1 \\
-1 & 0
\end{array}\right) .
$$

\section{Classification results}

The class of nonlocal operators (2) is invariant under projective transformations of the form

$$
\tilde{u}^{i}=\frac{l^{i}(u)}{l(u)}, \quad \tilde{g}=\frac{g}{l^{4}(u)}, \quad \tilde{w}=\frac{w}{l^{2}(u)},
$$

where $l_{i}, l$ are linear forms in the flat coordinates. Here $g=g_{i j}$ and $w=w_{i j}$ are the corresponding Monge metric and the skew-symmetric 2-form. This symmetry reflects the reciprocal invariance of nonlocal Hamiltonian formalism (2), thus generalising the analogous result known in the local case [11]. All our classification results are formulated modulo this equivalence.

Theorem 1 leads to a classification of nonlocal $n$-component Hamiltonian operators (2) based on normal forms of Monge metrics in dimensions $n=2$ and $n=3$ (due to the skew-symmetry of $w$ there exist no nonlocal operators of this type for $n=1$ ). 


\subsection{2-component case}

Theorem 2. In the 2-component case, every Monge metric gives rise to a Hamiltonian operator of type (2).

Proof. Every 2-component Monge metric is a quadratic form in the differentials $d p, d q$ and $p d q-q d p$. Thus, it can be represented as

$$
g=a(p d q-q d p)^{2}+2(p d q-q d p)(b d p+c d q)+\alpha d p^{2}+2 \beta d p d q+\gamma d q^{2}
$$

where $a, b, c, \alpha, \beta, \gamma$ are arbitrary constants. Every such metric gives rises to nonlocal operator (2) with

$$
g_{i j}=\left(\begin{array}{cc}
a q^{2}-2 b q+\alpha & -a p q+b p-c q+\beta \\
-a p q+b p-c q+\beta & a p^{2}+2 c p+\gamma
\end{array}\right), \quad w_{i j}=\frac{1}{\sqrt{\operatorname{det} g}}\left(\begin{array}{cc}
0 & 1 \\
-1 & 0
\end{array}\right) .
$$

This expression can be brought to normal form using affine transformations of $p$ and $q$. Case $a \neq 0$. Using translations of $p$ and $q$ we can set $b=c=0$. The rest depends on whether $\alpha \gamma-\beta^{2}$ is non-zero or not. In the non-zero case, using the remaining (complex) affine freedom we can also set $a=\alpha=\gamma=1, \beta=0$. This results in the metric

$$
g_{i j}=\left(\begin{array}{cc}
q^{2}+1 & -p q \\
-p q & p^{2}+1
\end{array}\right),
$$

which corresponds to the nonlocal Hamiltonian operator $A$ from Section 2. In the degenerate case $\alpha \gamma-\beta^{2}=0$ we can reduce the metric to the form

$$
g_{i j}=\left(\begin{array}{cc}
q^{2}+1 & -p q \\
-p q & p^{2}
\end{array}\right)
$$

which gives rise to the local Hamiltonian operator

$$
A=\partial_{x}\left(\begin{array}{cc}
\partial_{x} & \partial_{x} \frac{q}{p} \\
\frac{q}{p} \partial_{x} & \frac{q^{2}+1}{2 p^{2}} \partial_{x}+\partial_{x} \frac{q^{2}+1}{2 p^{2}}
\end{array}\right) \partial_{x} .
$$

Case $a=0$. Modulo affine transformations one can always assume $b=1, c=0$ (if $b=c=0$ we have a constant-coefficient metric corresponding to a constant-coefficient local operator). Using appropriate translations of $p$ and $q$ one can set $\alpha=\beta=0$. This results in the Monge metric

$$
g_{i j}=\left(\begin{array}{cc}
-2 q & p \\
p & \gamma
\end{array}\right)
$$

The case $\gamma \neq 0$ corresponds to nonlocal operator (2) with the skew-symmetric 2-form $w$ defined as

$$
w_{i j}=\frac{1}{\sqrt{-2 \gamma q-p^{2}}}\left(\begin{array}{cc}
0 & 1 \\
-1 & 0
\end{array}\right) .
$$

In the case $\gamma=0$ the above metric gives rise to the local Hamiltonian operator

$$
A=\partial_{x}\left(\begin{array}{cc}
0 & \partial_{x} \frac{1}{p} \\
\frac{1}{p} \partial_{x} & \frac{q}{p^{2}} \partial_{x}+\partial_{x} \frac{q}{p^{2}}
\end{array}\right) \partial_{x},
$$

which appeared as a Hamiltonian structure of Monge-Ampère equations [19], see also [11]. 


\subsection{3 -component case}

Every 3-component Monge metric $g$ can be written as a quadratic form in the 6 differentials $d u^{i}, u^{i} d u^{j}-u^{j} d u^{i}, i, j=1,2,3$. Let $Q$ denote the $6 \times 6$ symmetric matrix of this quadratic form. Let $P$ denote the $6 \times 6$ symmetric matrix corresponding to the quadratic Plücker relation,

$$
d u^{1}\left(u^{2} d u^{3}-u^{3} d u^{2}\right)+d u^{2}\left(u^{3} d u^{1}-u^{1} d u^{3}\right)+d u^{3}\left(u^{1} d u^{2}-u^{2} d u^{1}\right)=0 .
$$

Monge metrics are classified by their Segre types, that is, Jordan normal forms of the operator $Q P^{-1}$. In what follows we use the standard notation: thus, Segre type [123] indicates that the operator $Q P^{-1}$ has three Jordan blocks of sizes $1 \times 1,2 \times 2$ and $3 \times 3$, respectively. Additional round brackets indicate coincidences among the eigenvalues of these blocks: thus, [(12)3] indicates that the eigenvalue $\lambda_{1}$ of the first Jordan block coincides with the eigenvalue $\lambda_{2}$ of the second one, etc. We refer to [15, 10] for the list of normal forms of 3-component Monge metrics. All classification results are formulated modulo projective equivalence (6). In what follows we only present the Monge metric $g_{i j}$ and the skew-symmetric 2-form $w_{i j}$ (which uniquely specify the corresponding nonlocal operator (2); note that the 2 -form $w$ is defined up to an overall sign). The Theorem below provides a complete description of 3-component nonlocal operators (2) by going through the list of all Segre types and indicating particular allowed subcases that give rise to nonlocal operators. These are singled out by conditions (4). In each case we explicitly state the equation of the singular surface, det $g=0$, which is a quartic in $\mathbb{P}^{3}$ (possibly, reducible). It turns out that this quartic degenerates into a double quadric if and only if the operator is local.

Theorem 3. Modulo (complex) projective transformations (6) any nonlocal Hamiltonian operator (2) can be reduced to one of the following normal forms in the Segre classification:

1. Segre type [114]. Here the only allowed subcase is [(114)] which corresponds to the local operator defined by the metric $g^{(4)}$ from [11]:

$$
g_{i j}^{(4)}=\left(\begin{array}{ccc}
-2 u^{2} & u^{1} & 0 \\
u^{1} & 0 & 0 \\
0 & 0 & 1
\end{array}\right), \quad w_{i j}=0
$$

We have $\operatorname{det} g^{(4)}=-\left(u^{1}\right)^{2}$, the singular surface is a pair of double planes (one of them at infinity).

2. Segre type [123]. Here the only allowed subcase is [(123)] which corresponds to the local operator defined by the metric $g^{(5)}$ from [11]:

$$
g_{i j}^{(5)}=\left(\begin{array}{ccc}
-2 u^{2} & u^{1} & 1 \\
u^{1} & 1 & 0 \\
1 & 0 & 0
\end{array}\right), \quad w_{i j}=0
$$

We have $\operatorname{det} g^{(5)}=-1$, hence the singular surface is the quadruple plane at infinity. 
3. Segre type [222]. Here the only allowed subcase is [(222)] which corresponds to the local operator defined by the metric $g^{(6)}$ from [11]:

$$
g_{i j}^{(6)}=\left(\begin{array}{lll}
1 & 0 & 0 \\
0 & 1 & 0 \\
0 & 0 & 1
\end{array}\right), \quad w_{i j}=0 .
$$

We have $\operatorname{det} g^{(6)}=1$, the singular surface is the quadruple plane at infinity.

4. Segre type [15]. Here the only allowed subcase is [(15)] which gives rise to the nonlocal operator with the following metric $g$ and 2 -form $w$ :

$$
\begin{gathered}
g_{i j}=\left(\begin{array}{ccc}
0 & 1 & 2 u^{3} \\
1 & -2 u^{3} & u^{2} \\
2 u^{3} & u^{2} & -4 u^{1}
\end{array}\right) \\
w_{12}=0, \quad w_{31}=-\frac{1}{\sqrt{u^{1}+u^{2} u^{3}+2\left(u^{3}\right)^{3}}}, \quad w_{23}=\frac{u^{3}}{\sqrt{u^{1}+u^{2} u^{3}+2\left(u^{3}\right)^{3}}} .
\end{gathered}
$$

We have det $g=4 u^{1}+4 u^{2} u^{3}+8\left(u^{3}\right)^{3}$, the singular surface is a Cayley's ruled cubic and the plane at infinity.

5. Segre type [24]. Here the only allowed subcase is [(24)], which further splits into two projectively dual subcases. The first subcase gives rise to the nonlocal operator with the following metric $g$ and 2-form $w$ :

$$
\begin{gathered}
g_{i j}=\left(\begin{array}{ccc}
1 & 0 & u^{3} \\
0 & 1 & 0 \\
u^{3} & 0 & -2 u^{1}
\end{array}\right), \\
w_{12}=w_{23}=0, \quad w_{31}=\frac{1}{\sqrt{2 u^{1}+\left(u^{3}\right)^{2}}} .
\end{gathered}
$$

We have det $g=-2 u^{1}-\left(u^{3}\right)^{2}$, the singular surface is a quadratic cone and a double plane at infinity. The second subcase corresponds to

$$
\begin{gathered}
g_{i j}=\left(\begin{array}{ccc}
0 & 1 & 0 \\
1 & \left(u^{3}\right)^{2} & -u^{2} u^{3} \\
0 & -u^{2} u^{3} & 1+\left(u^{2}\right)^{2}
\end{array}\right), \\
w_{12}=w_{31}=0, \quad w_{23}=\frac{1}{\sqrt{1+\left(u^{2}\right)^{2}}} .
\end{gathered}
$$

We have det $g=-1-\left(u^{2}\right)^{2}$, the singular surface is a pair of planes and the double plane at infinity.

6. Segre type [33]. Here the only allowed subcase is [(33)] which gives rise to the nonlocal operator with the following metric $g$ and 2-form $w$ :

$$
\begin{gathered}
g_{i j}=\left(\begin{array}{ccc}
0 & 1 & 1 \\
1 & -2 u^{3} & u^{2}+u^{3} \\
1 & u^{2}+u^{3} & -2 u^{2}
\end{array}\right), \\
w_{12}=w_{31}=0, \quad w_{23}=\frac{2}{\sqrt{4 u^{2}+4 u^{3}}} .
\end{gathered}
$$


We have det $g=4\left(u^{2}+u^{3}\right)$, the singular surface is a plane and another triple plane at infinity.

7. Segre type [6]. This case does not correspond to any Hamiltonian operator.

8. Segre type [1122]. There are 3 allowed subcases:

- subcase [(11)22] with the additional constraint $2 \lambda_{1}=\lambda_{3}+\lambda_{4}$. This gives rise to the nonlocal operator with the following metric $g$ and 2 -form $w$ :

$$
\begin{gathered}
g_{i j}=\left(\begin{array}{ccc}
1 & -2 \lambda u^{3} & \lambda u^{2} \\
-2 \lambda u^{3} & 4 & \lambda u^{1} \\
\lambda u^{2} & \lambda u^{1} & 0
\end{array}\right), \\
w_{12}=0, \quad w_{23}=\frac{\lambda^{2} u^{1}}{\sqrt{\operatorname{det} g}}, \quad w_{31}=\frac{\lambda^{2} u^{2}}{\sqrt{\operatorname{det} g}},
\end{gathered}
$$

where $\lambda=\lambda_{3}-\lambda_{4}$. We have $\operatorname{det} g=-\lambda^{2}\left(u^{1}\right)^{2}-4 \lambda^{2}\left(u^{2}\right)^{2}-4 \lambda^{3} u^{1} u^{2} u^{3}$, the singular surface is a cubic and the plane at infinity.

- subcase [11(22)] with the additional constraint $\lambda_{1}+\lambda_{2}=2 \lambda_{3}$. This gives rise to the nonlocal operator with the following metric $g$ and 2 -form $w$ :

$$
\begin{gathered}
g_{i j}=\left(\begin{array}{ccc}
1+\lambda\left(u^{2}\right)^{2} & -\lambda u^{1} u^{2} & 0 \\
-\lambda u^{1} u^{2} & 4+\lambda\left(u^{1}\right)^{2} & 0 \\
0 & 0 & \lambda
\end{array}\right), \\
w_{12}=\frac{2 \lambda}{\sqrt{\operatorname{det} g}}, \quad w_{31}=w_{23}=0
\end{gathered}
$$

where $\lambda=\lambda_{1}-\lambda_{2}$. We have det $g=4 \lambda+\lambda^{2}\left(u^{1}\right)^{2}+4 \lambda^{2}\left(u^{2}\right)^{2}$, the singular surface is a quadratic cone and the double plane at infinity.

- subcase [1(12)2], with the additional constraint $3 \lambda_{2}=2 \lambda_{4}+\lambda_{1}$. This gives rise to the nonlocal operator with the following metric $g$ and 2 -form $w$ :

$$
\begin{gathered}
g_{i j}=\left(\begin{array}{ccc}
1+\lambda\left(u^{2}\right)^{2} & \lambda\left(-u^{1} u^{2}+u^{3}\right) & \lambda u^{2} \\
\lambda\left(-u^{1} u^{2}+u^{3}\right) & 4+\lambda\left(u^{1}\right)^{2} & -2 \lambda u^{1} \\
\lambda u^{2} & -2 \lambda u^{1} & \lambda
\end{array}\right), \\
w_{12}=\frac{\lambda \sqrt{\lambda} u^{1}}{\sqrt{-\operatorname{det} g}}, \quad w_{23}=0, \quad w_{31}=-\frac{\lambda \sqrt{\lambda}}{\sqrt{-\operatorname{det} g}},
\end{gathered}
$$

where $\lambda=\lambda_{1}-\lambda_{2}$. We have det $g=4 \lambda-3 \lambda^{2}\left(u^{1}\right)^{2}-4 \lambda^{3}\left(u^{1} u^{2}\right)^{2}+\lambda^{3}\left(u^{3}-u^{1} u^{2}\right)^{2}$, the singular surface is an irreducible quartic.

9. Segre type [1113]. There are 2 essentially different allowed subcases: 
- subcase [11(13)], with the additional constraint $2 \lambda_{3}=\lambda_{1}+\lambda_{2}$. This gives rise to the nonlocal operator with the following metric $g$ and 2-form $w$ :

$$
\begin{gathered}
g_{i j}=\left(\begin{array}{ccc}
2 u^{3}+\lambda\left(u^{2}\right)^{2} & -1-\lambda u^{1} u^{2} & -u^{1} \\
-1-\lambda u^{1} u^{2} & \lambda\left(u^{1}\right)^{2} & 0 \\
-u^{1} & 0 & \lambda
\end{array}\right), \\
w_{12}=\frac{\lambda}{\sqrt{-\operatorname{det} g}}, \quad w_{23}=0, \quad w_{31}=\frac{\lambda u^{1}}{\sqrt{-\operatorname{det} g}},
\end{gathered}
$$

where $\lambda=\lambda_{1}-\lambda_{2}$. We have det $g=-\lambda-2 \lambda^{2} u^{1} u^{2}+2 \lambda^{2} u^{3}\left(u^{1}\right)^{2}-\lambda\left(u^{1}\right)^{4}$, the singular surface is an irreducible quartic.

- subcase [(11)13], with the additional constraint $4 \lambda_{1}=\lambda_{3}+3 \lambda_{4}$. This gives rise to the nonlocal operator with the following metric $g$ and 2-form $w$ :

$$
\begin{gathered}
g_{i j}=\left(\begin{array}{ccc}
2 u^{3}-2 \lambda\left(u^{3}\right)^{2} & -1-2 \lambda u^{3} & -u^{1}+\lambda u^{2}+2 \lambda u^{1} u^{3} \\
-1-2 \lambda u^{3} & -2 \lambda & \lambda u^{1} \\
-u^{1}+\lambda u^{2}+2 \lambda u^{1} u^{3} & \lambda u^{1} & -2 \lambda\left(u^{1}\right)^{2}
\end{array}\right), \\
\omega_{12}=0, \quad \omega_{23}=\frac{\sqrt{3} \lambda^{2} u^{1}}{\sqrt{-\operatorname{det} g}}, \quad \omega_{31}=\frac{\sqrt{3} \lambda^{2} u^{2}}{\sqrt{-\operatorname{det} g}}
\end{gathered}
$$

where $\lambda=2\left(\lambda_{1}-\lambda_{4}\right)$. We have det $g=2 \lambda\left(\lambda^{2}\left(u^{1}\right)^{2}\left(u^{3}\right)^{2}+2 \lambda^{2} u^{1} u^{2} u^{3}+\lambda^{2}\left(u^{2}\right)^{2}+\right.$ $\left.3 \lambda\left(u^{1}\right)^{2} u^{3}-3 \lambda u^{1} u^{2}+3\left(u^{1}\right)^{2}\right)$, the singular surface is an irreducible quartic.

10. Segre type [11112]. There are 2 essentially different allowed subcases:

- subcase $[111(12)]$, with the additional constraint $\lambda_{1}+\lambda_{2}+\lambda_{3}=3 \lambda_{4}$. This gives rise to the nonlocal operator with the following metric $g$ and 2-form $w$ :

$$
\begin{aligned}
& g_{i j}=\left(\begin{array}{ccc}
1+\lambda\left(u^{2}\right)^{2}+\mu\left(u^{3}\right)^{2} & -\lambda\left(u^{3}+u^{1} u^{2}\right) & -\lambda u^{2}-\mu u^{1} u^{3} \\
-\lambda\left(u^{3}+u^{1} u^{2}\right) & \lambda\left(u^{1}\right)^{2}+\mu & 2 \lambda u^{1} \\
-\lambda u^{2}-\mu u^{1} u^{3} & 2 \lambda u^{1} & \lambda+\mu\left(u^{1}\right)^{2}
\end{array}\right), \\
& w_{12}=\sqrt{\frac{\lambda\left(\mu^{2}-\lambda^{2}\right)}{\operatorname{det} g}} u^{1}, \quad w_{23}=0, \quad w_{31}=\sqrt{\frac{\lambda\left(\mu^{2}-\lambda^{2}\right)}{\operatorname{det} g}}
\end{aligned}
$$

where $\lambda=\lambda_{1}-\lambda_{4}$ and $\mu=\lambda_{3}-\lambda_{2}$. We have

$$
\begin{aligned}
\operatorname{det} g= & -\lambda^{3}\left(u^{1} u^{2}\right)^{2}+2 \lambda^{3} u^{1} u^{2} u^{3}-\lambda^{3}\left(u^{3}\right)^{2}-3 \lambda^{2}\left(u^{1}\right)^{2} \\
& +\lambda \mu^{2}\left(u^{1} u^{2}\right)^{2}-2 \lambda \mu^{2} u^{1} u^{2} u^{3}+\lambda \mu^{2}\left(u^{3}\right)^{2}+\lambda \mu\left(u^{1}\right)^{4}+\lambda \mu+\mu^{2}\left(u^{1}\right)^{2}
\end{aligned}
$$

the singular surface is an irreducible quartic. Note that the additional constraint $\lambda^{2}=\mu^{2}$ leads to a local operator. 
- subcase [(11)112], with the additional constraint $4 \lambda_{1}=\lambda_{3}+\lambda_{4}+2 \lambda_{5}$. This gives rise to the nonlocal operator with the following metric $g$ and 2-form $w$ :

$$
\begin{gathered}
g_{i j}=\left(\begin{array}{ccc}
1+\mu\left(u^{3}\right)^{2} & -2 \beta u^{3} & \beta u^{2}-\mu u^{1} u^{3} \\
-2 \beta u^{3} & \mu & \beta u^{1} \\
\beta u^{2}-\mu u^{1} u^{3} & \beta u^{1} & \mu\left(u^{1}\right)^{2}
\end{array}\right), \\
w_{12}=0, \quad w_{23}=\sqrt{\frac{\beta^{2}\left(\beta^{2}-\mu^{2}\right)}{\operatorname{det} g}} u^{1}, \quad w_{31}=\sqrt{\frac{\beta^{2}\left(\beta^{2}-\mu^{2}\right)}{\operatorname{det} g}} u^{2},
\end{gathered}
$$

where $\mu=\lambda_{3}-\lambda_{4}$ and $\beta=2\left(\lambda_{1}-\lambda_{5}\right)$. We have

$$
\operatorname{det} g=2 \lambda\left(\lambda^{2}\left(u^{1} u^{3}\right)^{2}+2 \lambda^{2} u^{1} u^{2} u^{3}+\lambda^{2}\left(u^{2}\right)^{2}+3 \lambda\left(u^{1}\right)^{2} u^{3}-3 \lambda u^{1} u^{2}+3\left(u^{1}\right)^{2}\right),
$$

the singular surface is an irreducible quartic. Note that the additional constraint $\beta^{2}=\mu^{2}$ leads to a local operator.

11. Segre type [111111]. Up to relabelling of the eigenvalues, there is essentially only one allowed subcase, namely [1111(11)], with the additional constraint $\lambda_{1}+\lambda_{2}+\lambda_{3}+$ $\lambda_{4}=4 \lambda_{5}$. This gives rise to the nonlocal operator with the following metric $g$ and 2-form $w$ :

$$
\begin{gathered}
g_{i j}=\left(\begin{array}{ccc}
a_{3}\left(u^{2}\right)^{2}+a_{2}\left(u^{3}\right)^{2} & -a_{3} u^{1} u^{2}+\alpha u^{3} & -a_{2} u^{1} u^{3}+\alpha u^{2} \\
-a_{3} u^{1} u^{2}+\alpha u^{3} & a_{2}+a_{3}\left(u^{1}\right)^{2} & -2 \alpha u^{1} \\
-a_{2} u^{1} u^{3}+\alpha u^{2} & -2 \alpha u^{1} & a_{3}+a_{2}\left(u^{1}\right)^{2}
\end{array}\right), \\
w_{12}=\sqrt{\frac{\left(a_{2}^{2}-\alpha^{2}\right)\left(a_{3}^{2}-\alpha^{2}\right)}{\operatorname{det} g}} u^{3}, \quad w_{23}=0, \quad w_{31}=\sqrt{\frac{\left(a_{2}^{2}-\alpha^{2}\right)\left(a_{3}^{2}-\alpha^{2}\right)}{\operatorname{det} g}} u^{2},
\end{gathered}
$$

where $a_{2}=\lambda_{3}-\lambda_{4}, a_{3}=\lambda_{1}-\lambda_{2}$ and $\alpha=2 \lambda_{5}-\lambda_{3}-\lambda_{4}$. We have

$$
\begin{aligned}
\operatorname{det} g=a_{2}^{2} a_{3}( & \left.u^{1} u^{2}\right)^{2}+a_{2}^{2} a_{3}\left(u^{3}\right)^{2}+2 a_{2}^{2} \alpha u^{1} u^{2} u^{3} \\
+ & +a_{2} a_{3}^{2}\left(u^{1} u^{3}\right)^{2}+a_{2} a_{3}^{2}\left(u^{2}\right)^{2}-a_{2} \alpha^{2}\left(u^{1} u^{3}\right)^{2}-a_{2} \alpha^{2}\left(u^{2}\right)^{2} \\
& +2 a_{3}^{2} \alpha u^{1} u^{2} u^{3}-a_{3} \alpha^{2}\left(u^{1} u^{2}\right)^{2}-a_{3} \alpha^{2}\left(u^{3}\right)^{2}-4 \alpha^{3} u^{1} u^{2} u^{3},
\end{aligned}
$$

the singular surface is an irreducible quartic. Note that the additional constraints $\alpha_{2}^{2}=\alpha^{2}$ or $\alpha_{2}^{2}=\alpha^{2}$ lead to local operators.

\section{Nonlocal operators via Dirac reduction}

Let us consider an $(n+1)$-component local third-order Hamiltonian operator

$$
A^{I J}=\partial_{x}\left(G^{I J}(u) \partial_{x}+C_{K}^{I J}(u) u_{x}^{K}\right) \partial_{x}
$$

represented in the flat coordinates $u^{0}, \ldots, u^{n}$. In this section we will calculate the Dirac reduction of this operator to a hyperplane in the $u$-coordinates. Without any loss of generality one can assume that this hyperplane is given by the equation $u^{0}=0$. In what follows we use the following convention for the small and capital indices: $i, j, k \in$ $\{1, \ldots, n\}, I, J, K \in\{0, \ldots, n\}$. 
Theorem 4. Dirac reduction of local Hamiltonian operator (7) to the hyperplane $u^{0}=0$ is given by nonlocal operator (2) where $g_{i j}=G_{i j}, c_{i j k}=C_{i j k}, w_{i j}=\frac{C_{i j}^{0}}{\sqrt{G^{00}}}$.

Proof. We find it more convenient to work in potential coordinates $b^{K}$ defined as $u^{K}=b_{x}^{K}$. In these coordinates operator (7) takes first-order form,

$$
A^{I J}=-G^{I J}\left(b_{x}\right) \partial_{x}-C_{K}^{I J}\left(b_{x}\right) b_{x x}^{K} .
$$

Its Dirac reduction $\tilde{A}$ to the hyperplane $b^{0}=0$ is defined by the formula

$$
\tilde{A}^{i j}=A^{i j}-A^{i 0}\left(A^{00}\right)^{-1} A^{0 j} .
$$

Since $A^{00}=-G^{00} \partial_{x}-C_{K}^{00} b_{x x}^{K}$ and $G_{, K}^{00}=2 C_{K}^{00}$ we obtain

$$
A^{00}=-G^{00} \partial_{x}-\frac{1}{2} G_{, K}^{00} b_{x x}^{K}=-G^{00} \partial_{x}-\frac{1}{2}\left(G^{00}\right)_{x}=-\sqrt{G^{00}} \partial_{x} \sqrt{G^{00}},
$$

so that

$$
\left(A^{00}\right)^{-1}=-\frac{1}{\sqrt{G^{00}}} \partial_{x}^{-1} \frac{1}{\sqrt{G^{00}}} .
$$

Thus,

$$
\tilde{A}^{i j}=A^{i j}+A^{i 0} \frac{1}{\sqrt{G^{00}}} \partial_{x}^{-1} \frac{1}{\sqrt{G^{00}}} A^{0 j},
$$

where

$$
A^{i j}=-G^{i j} \partial_{x}-C_{k}^{i j} b_{x x}^{k},
$$

note that $b^{0}=0$. Explicitly, this gives

$$
\tilde{A}^{i j}=-G^{i j} \partial_{x}-C_{k}^{i j} b_{x x}^{k}+\left(G^{i 0} \partial_{x}+C_{k}^{i 0} b_{x x}^{k}\right) \frac{1}{\sqrt{G^{00}}} \partial_{x}^{-1} \frac{1}{\sqrt{G^{00}}}\left(G^{0 j} \partial_{x}+C_{k}^{0 j} b_{x x}^{k}\right) .
$$

This expression can be rewritten in the form

$$
\tilde{A}^{i j}=-g^{i j} \partial_{x}-c_{k}^{i j} b_{x x}^{k}-w_{k}^{i} b_{x x}^{k} \partial_{x}^{-1} w_{m}^{j} b_{x x}^{m}
$$

which reduces to (2) in the original variables $u^{i}=b_{x}^{i}$. Here

$$
\begin{gathered}
g^{i j}=G^{i j}-\frac{G^{i 0} G^{0 j}}{G^{00}}, \quad c_{k}^{i j}=C_{k}^{i j}-\frac{G^{i 0} C_{k}^{0 j}+C_{k}^{i 0} G^{0 j}}{G^{00}}+\frac{G^{i 0} G^{0 j} G_{, k}^{00}}{2\left(G^{00}\right)^{2}}, \\
w_{k}^{i}=\frac{1}{\sqrt{G^{00}}} C_{k}^{i 0}-\frac{1}{2} \frac{G^{i 0} G_{, k}^{00}}{\left(G^{00}\right)^{3 / 2}} .
\end{gathered}
$$

The formula for $g^{i j}$ precisely means that $g_{i j}=G_{i j}$. The formula for $c_{i j k}$ follows from the fact the $c$ is determined by $g$ (see Remark at the end of Section 1). It remains to prove that the expression for $w_{k}^{i}$ is equivalent to the formula $w_{i j}=\frac{C_{i j}^{0}}{\sqrt{G^{00}}}$. Since

$$
C_{k}^{i 0}=G^{i 0} C_{0 k}^{0}+G^{i m} C_{m k}^{0}
$$


we obtain

$$
w_{k}^{i}=\frac{1}{\sqrt{G^{00}}}\left[G^{i 0} C_{0 k}^{0}+G^{i m} C_{m k}^{0}\right]-\frac{1}{2} \frac{G^{i 0}}{\left(G^{00}\right)^{3 / 2}} G_{, k}^{00} .
$$

Taking into account

$$
G^{i k}=g^{i k}+\frac{G^{i 0} G^{0 k}}{G^{00}}
$$

this gives

$$
w_{k}^{i}=\frac{1}{\left(G^{00}\right)^{3 / 2}}\left[G^{00} G^{i 0} C_{0 k}^{0}+\left(G^{00} g^{i m}+G^{i 0} G^{0 m}\right) C_{m k}^{0}-\frac{1}{2} G^{i 0} G_{, k}^{00}\right] .
$$

Using the derivative of the inverse matrix,

$$
G_{, K}^{I J}=-G^{I P} G_{P Q, K} G^{Q J}
$$

we obtain

$$
G_{, k}^{00}=-\left(G^{00}\right)^{2} G_{00, k}-2 G^{00} G^{0 m} G_{0 m, k}-G^{0 m} G^{0 s} g_{m s, k} .
$$

Taking into account

$$
C_{0 k}^{0}=G^{00} C_{00 k}+G^{0 m} C_{m 0 k}, \quad C_{i k}^{0}=G^{00} C_{0 i k}+G^{0 s} C_{s i k},
$$

on simplification we obtain

$$
w_{k}^{i}=\frac{g^{i m} C_{m k}^{0}}{\sqrt{G^{00}}}
$$

which is equivalent to the required formula $w_{i j}=\frac{C_{i j}^{0}}{\sqrt{G^{00}}}$.

\section{Skew-symmetry conditions and Jacobi identities: proof of Theorem 1}

The standard way to calculate skew-symmetry conditions and Jacobi identities is based on the Gelfand-Dorfman approach [14]. In this Section we utilise an alternative technique based on the theory of Poisson vertex algebras [2,3] which gives a completely algebraic approach to local and nonlocal Hamiltonian operators. This is achieved by considering the differential algebra corresponding, in the theory of the formal calculus of variations, to the densities of local functionals - usually, the space of differential polynomials or some extension thereof. More precisely, any Poisson vertex algebra defines a Poisson bracket on the space of local functionals and an action of the space of local functionals on the space of their densities: such objects are, equivalently, defined by Hamiltonian operators. Conversely, a Hamiltonian operator on the space of densities, either differential or pseudodifferential (under some additional technical hypotheses), defines a Poisson vertex algebra.

Definition 1. A (nonlocal) Poisson vertex algebra (PVA) is a differential algebra $(\mathcal{A}, \partial)$ endowed with a derivation $\partial$ and a bilinear operation $\left\{\cdot{ }_{\lambda} \cdot\right\}: \mathcal{A} \otimes \mathcal{A} \rightarrow \mathbb{R}\left(\left(\lambda^{-1}\right)\right) \otimes \mathcal{A}$ called $a$ (nonlocal) $\lambda$ bracket, satisfying the following set of properties: 
1. $\left\{\partial f_{\lambda} g\right\}=-\lambda\left\{f_{\lambda} g\right\}$ (left sesquilinearity),

2. $\left\{f_{\lambda} \partial g\right\}=(\lambda+\partial)\left\{f_{\lambda} g\right\}$ (right sesquilinearity),

3. $\left\{f_{\lambda} g h\right\}=\left\{f_{\lambda} g\right\} h+\left\{f_{\lambda} h\right\}$ (left Leibnitz property),

4. $\left\{f g_{\lambda} h\right\}=\left\{f_{\lambda+\partial} h\right\} g+\left\{g_{\lambda+\partial} h\right\} f$ (right Leibnitz property),

5. $\left\{g_{\lambda} f\right\}=-\rightarrow\left\{f_{-\lambda-g} g\right\}$ (PVA skew-symmetry),

6. $\left\{f_{\lambda}\left\{g_{\mu} h\right\}\right\}-\left\{g_{\mu}\left\{f_{\lambda} h\right\}\right\}=\left\{\left\{f_{\lambda} g\right\}_{\lambda+\mu} h\right\}$ (PVA-Jacobi identity).

Let us denote

$$
\left\{f_{\lambda} g\right\}=\sum_{s \leq S} C_{s}(f, g) \lambda^{s} .
$$

The expansion of the bracket in $\lambda$ is bounded by $0 \leq s \leq S$ for local PVAs and is not bounded from below for nonlocal PVAs. Using the expansion, the expressions on the RHS of Property 4 are to be understood as $\left\{f_{\lambda+\partial} g\right\} h=\sum C_{s}(f, g)(\lambda+\partial)^{s} h=$ $\sum_{s} \sum_{t} C_{s}\left(\begin{array}{l}s \\ t\end{array}\right) \partial^{t} h \lambda^{s-t}$, while the RHS of Property 5 reads $\rightarrow\left\{f_{-\lambda-\partial} g\right\}=\sum_{s}(-\lambda-\partial)^{s} C_{s}(f, g)$.

For the case of nonlocal PVAs, it should be noted that the three terms of PVAJacobi identity do not necessarily belong to the same space, because of the double infinite expansion of the brackets (in terms of $(\lambda, \mu),(\mu, \lambda)$ and $(\lambda, \lambda+\mu)$, respectively). A bracket is said to be admissible if all the three terms can be (not uniquely) expanded as

$$
\left\{f_{\lambda}\left\{g_{\mu} h\right\}\right\}=\sum_{m \leq M} \sum_{n \leq N} \sum_{p \leq 0} a_{m, n, p} \lambda^{m} \mu^{n}(\lambda+\mu)^{p},
$$

and only admissible brackets can define a nonlocal PVA. We denote the space where the PVA-Jacobi identity of admissible brackets takes values as $V_{\lambda, \mu}$. This space can be decomposed by the total degree $d$ in $(\lambda, \mu, \lambda+\mu)$; finally, elements of each homogeneous component $V_{\lambda, \mu}^{(d)}$ can be uniquely expressed in the basis [3]

$$
\begin{array}{rl}
\lambda^{i} \mu^{d-i} & i \in \mathbb{Z}, \\
\lambda^{d+i}(\lambda+\mu)^{-i} & i=\{1,2, \ldots\} .
\end{array}
$$

The main advantage of PVAs is the existence of a closed and explicit formula to compute the $\lambda$ bracket of any two elements of $\mathcal{A}$, in terms of the bracket between the generators of $\mathcal{A}$. Such a formula is called the master formula and reads

$$
\left\{f_{\lambda} g\right\}=\sum_{i, j=1}^{n} \sum_{l \geq 0} \sum_{m \geq 0} \frac{\partial g}{\partial u_{(m)}^{j}}(\lambda+\partial)^{m}\left\{u_{\lambda+\partial}^{i} u^{j}\right\}(-\lambda-\partial)^{l} \frac{\partial f}{\partial u_{(l)}^{i}}
$$

where $n$ is the number of generators of $\mathcal{A}$ and $u_{(l)}^{i}$ denotes the $l$-th jet coordinate $\left(\partial u_{(l)}^{i}=\right.$ $\left.u_{(l+1)}^{i}\right)$.

Given a Hamiltonian operator $P^{i j}(\partial)$, the $\lambda$ bracket of the corresponding PVA is obtained by setting the bracket between the generators equal the transpose of the symbol of the operator, $\left\{u_{\lambda}^{i} u^{j}\right\}=P^{j i}(\lambda)$. The strategy of our proof consists in obtaining the $\lambda$ 
bracket corresponding to the candidate Hamiltonian operator of third order, and requiring that it must satisfy the skew-symmetry and PVA-Jacobi properties. They are equivalent [2] to the skew-symmetry and the Jacobi identity for the Poisson bracket defined by the operator - hence the conditions that we derive are the conditions for the operator to be Hamiltonian.

\subsection{The $\lambda$ bracket}

The operator $A$ defined by (2) corresponds to the $\lambda$ bracket of the form

$$
\left\{u_{\lambda}^{i} u^{j}\right\}=(\lambda+\partial)\left(g^{j i} \lambda+c_{l}^{j i} u_{x}^{l}+w_{l}^{j} u_{x}^{l}(\lambda+\partial)^{-1} w_{m}^{i} u_{x}^{m}\right) \lambda .
$$

For convenience, we express the $\lambda$ bracket (9) in potential coordinates $v_{x}^{i}=u^{i}$ where it takes the form $-\left\{u_{\lambda}^{i} u^{j}\right\}=\left\{v_{\lambda}^{i} v^{j}\right\}^{\prime}=\left\{v_{\lambda}^{i} v^{j}\right\}_{L}+\left\{v_{\lambda}^{i} v^{j}\right\}_{N}$, with

$$
\left\{v_{\lambda}^{i} v^{j}\right\}_{L}=g^{j i} \lambda+c_{k}^{j i} v_{2 x}^{k}, \quad\left\{v_{\lambda}^{i} v^{j}\right\}_{N}=w_{l}^{j} v_{2 x}^{l}(\lambda+\partial)^{-1} w_{m}^{i} v_{2 x}^{m}
$$

and all the functions depending on $v_{x}^{i}$ only. We choose to decompose the bracket in its local part $\left\{v_{\lambda}^{i} v^{j}\right\}_{L}$ and its nonlocal part $\left\{v_{\lambda}^{i} v^{j}\right\}_{N}$. The nonlocal part is admissible, being a ratio of local $\lambda$ brackets [3].

\subsection{The skew-symmetry condition}

The condition of skew-symmetry is equivalent to the conditions

$$
g^{i j}=g^{j i}, \quad g_{, k}^{i j}=c_{k}^{i j}+c_{k}^{j i} .
$$

Indeed, for the local part we have

$$
\left\{v_{\lambda}^{i} v^{j}\right\}_{L}=g^{j i} \lambda+c_{k}^{j i} v_{2 x}^{k}=-{ }_{\rightarrow}\left\{v_{-\lambda-\partial}^{j} v^{i}\right\}_{L}=g^{i j} \lambda+\partial_{k} g^{i j} v_{2 x}^{k}-c_{k}^{i j} v_{2 x}^{k} .
$$

The two identities are the coefficients of $\lambda$ and of $v_{2 x}^{k}$ in both sides of the equation.

The nonlocal part is skew-symmetric by construction. This can be shown by taking the formal series expansion of $(\lambda+\partial)^{-1}$, substituting the powers of $\lambda$ with $(-\lambda-\partial)$ and taking a double expansion. However this procedure can be replaced by a much simpler one: in the nonlocal part of the bracket, $w_{l}^{j} v_{2 x}^{l}(\lambda+\partial)^{-1} w_{m}^{i} v_{2 x}^{m}$, the total derivative in the parenthesis acts only on terms on its right-hand side. For shorthand, we can write $(\lambda+\partial)^{-1}$ as $\left(\lambda+\partial^{(i)}\right)^{-1}$ where the superscript means that it acts on $w_{m}^{i} v_{2 x}^{m}$. By doing this, the actual position of the operator $(\lambda+\partial)^{-1}$ becomes irrelevant. Thus,

$$
\left\{v_{\lambda}^{i} v^{j}\right\}_{N}=\left(\lambda+\partial^{(i)}\right)^{-1}\left(w_{l}^{j} v_{2 x}^{l}\right)\left(w_{m}^{i} v_{2 x}^{m}\right)
$$

On the other hand, the total derivative in the definition of skew-symmetry acts on all the bracket, namely it can be interpreted as $\partial^{(i)}+\partial^{(j)}$. This means that

$$
\rightarrow\left\{v_{-\lambda-\partial}^{j} v^{i}\right\}=\left(-\lambda-\partial^{(i)}-\partial^{(j)}+\partial^{(j)}\right)^{-1}\left(w_{l}^{j} v_{2 x}^{l}\right)\left(w_{m}^{i} v_{2 x}^{m}\right),
$$

from which the skew-symmetry easily follows. 


\subsection{The PVA-Jacobi identity}

The PVA-Jacobi identity for the bracket (9) splits into four parts, when taking into account local and nonlocal parts separately. Let us adopt the shorthand notation

$$
\begin{gathered}
T_{P, Q}^{i j k}(\lambda, \mu):=\left\{u_{\lambda}^{i}\left\{u_{\mu}^{j} u^{k}\right\}_{P}\right\}_{Q}, \\
J^{i j k}(A, B):=T_{A, B}^{i j k}(\lambda, \mu)-T_{A, B}^{j i k}(\mu, \lambda)+T_{A, B}^{k i j}(-\lambda-\mu-\partial, \lambda),
\end{gathered}
$$

for the terms of the PVA-Jacobi identity, where $(P, Q)$ denote different $\lambda$ brackets and the last term in $J^{i j k}$ should be understood as $\rightarrow\left\{u_{-\lambda-\mu-\partial}^{k}\left\{u_{\lambda}^{i} u^{j}\right\}_{A}\right\}_{B}$, using the skew-symmetry property of the $\lambda$ bracket. The PVA-Jacobi identity can then be written as

$$
J^{i j k}(A, A)=0
$$

By the linearity of the bracket, the PVA-Jacobi identity reads

$$
J^{i j k}(A, A)=J^{i j k}(L, L)+J^{i j k}(L, N)+J^{i j k}(N, L)+J^{i j k}(N, N)=0 .
$$

The computation of $J^{i j k}(L, L)$ is a straightforward application of the master formula (8).

The expressions involving nonlocal terms live in the space $V_{\lambda, \mu}$ whose homogeneous components have the basis $\left(\lambda^{i} \mu^{d-i}, \lambda^{d+j}(\lambda+\mu)^{-j}\right)$ with $i \in \mathbb{Z}$ and $j \in \mathbb{Z}_{+}$. We choose to isolate the nonlocal coefficients of the form $P[\mu]\left[(\lambda+\partial)^{-1} w_{s}^{i} v_{2 x}^{s}\right], P[\lambda]\left[(\mu+\partial)^{-1} w_{s}^{j} v_{2 x}^{s}\right]$ and $w_{s}^{k} v_{2 x}^{s}(\lambda+\mu+\partial)^{-1} P[\lambda]$ where $P[\nu]$ are polynomials in the formal parameter $\nu$ with differential polynomials as coefficients. Expanding $(\nu+\partial)^{-1} f$ by $\sum_{k \geq 0}(-1)^{k} \nu^{-1-k} \partial^{k} f$ in the former two expressions we obtain elements in the subspace whose basis is $\lambda^{i} \mu^{d-i}$; doing the same in the latter produces elements in the subspace $(\lambda+\mu)^{-j} \lambda^{d+j}$. In fact, they give an infinite number of coefficient, but it is apparent that the vanishing of the terms for $k=0$ (corresponding to $\lambda^{-1}, \mu^{-1}$ and $(\lambda+\mu)^{-1}$, respectively) is necessary and sufficient for the vanishing of all the expansion (because the further elements of the expansion have a different total degree $d$, so correspond to other elements of the basis).

To explicitly show how we compute the $\lambda$ bracket for nonlocal PVAs and how we express the terms of the PVA-Jacobi identity in the basis of $V_{\lambda, \mu}$, we demonstrate the full computation for the term $T_{N, L}^{j i k}(\mu, \lambda)$, i.e. the second summand of $J^{i j k}(N, L)$. We have

$$
\left\{v_{\mu}^{j}\left\{v_{\lambda}^{i} v^{k}\right\}_{N}\right\}_{L}=\left\{v_{\mu}^{j} w_{m}^{k} v_{2 x}^{m}(\lambda+\partial)^{-1} w_{n}^{i} v_{2 x}^{n}\right\}_{L} .
$$

By Leibniz's property this expression equals

$$
\left[(\lambda+\partial)^{-1} w_{n}^{i} v_{2 x}^{n}\right]\left\{v_{\mu}^{j} w_{m}^{k} v_{2 x}^{m}\right\}_{L}+w_{m}^{k} v_{2 x}^{m}\left\{v_{\mu}^{j}(\lambda+\partial)^{-1} w_{n}^{i} v_{2 x}^{n}\right\}_{L},
$$

(the square brackets remind us that the pseudodifferential operator does not act outside them). Using sesquilinearity on the second summand we obtain

$$
\begin{aligned}
& {\left[(\lambda+\partial)^{-1} w_{n}^{i} v_{2 x}^{n}\right]\left\{v_{\mu}^{j} w_{m}^{k} v_{2 x}^{m}\right\}_{L}+w_{m}^{k} v_{2 x}^{m}(\lambda+\mu+\partial)^{-1}\left\{v_{\mu}^{j} w_{n}^{i} v_{2 x}^{n}\right\}_{L}} \\
& =\left[(\lambda+\partial)^{-1} w_{n}^{i} v_{2 x}^{n}\right]\left(w_{m, l}^{k} v_{2 x}^{m}(\mu+\partial)\left(g^{l j} \mu+c_{s}^{l j} v_{2 x}^{s}\right)+w_{l}^{k}(\mu+\partial)^{2}\left(g^{l j} \mu+c_{s}^{l j} v_{2 x}^{s}\right)\right) \\
& +w_{m}^{k} v_{2 x}^{m}(\lambda+\mu+\partial)^{-1}\left(w_{n, l}^{i} v_{2 x}^{n}(\mu+\partial)\left(g^{l j} \mu+c_{s}^{l j} v_{2 x}^{s}\right)+w_{l}^{i}(\mu+\partial)^{2}\left(g^{l j} \mu+c_{s}^{l j} v_{2 x}^{s}\right)\right) .
\end{aligned}
$$


The first line of the expression is of the form $\left[(\lambda+\partial)^{-1} w_{n}^{i} v_{2 x}^{n}\right] P[\mu]$, so it is enough to expand $P$. On the other hand, the second line of the expression is of the form $w_{s}^{k} v_{2 x}^{s}(\lambda+$ $\mu+\partial)^{-1} P[\mu]$ which cannot be immediately expanded in the basis of $V_{\lambda, \mu}$. Consider for instance

$$
w_{m}^{k} v_{2 x}^{m}(\lambda+\mu+\partial)^{-1}\left(w_{n, l}^{i} v_{2 x}^{n}(\mu+\partial)\left(g^{l j} \mu+c_{s}^{l j} v_{2 x}^{s}\right)\right) .
$$

We proceed by replacing $(\mu+\partial)$ with $(\lambda+\mu+\partial)$, then moving it to the right of its inverse to make them cancel out:

$$
\begin{gathered}
w_{m}^{k} v_{2 x}^{m}(\lambda+\mu+\partial)^{-1}\left(w_{n, l}^{i} v_{2 x}^{n}(\mu+\partial)\left(g^{l j} \mu+c_{s}^{l j} v_{2 x}^{s}\right)\right)= \\
w_{m}^{k} v_{2 x}^{m}(\lambda+\mu+\partial)^{-1}(\lambda+\mu+\partial)\left(w_{n, l}^{i} v_{2 x}^{n}\left(g^{l j} \mu+c_{s}^{l j} v_{2 x}^{s}\right)\right) \\
-w_{m}^{k} v_{2 x}^{m}(\lambda+\mu+\partial)^{-1}\left(\lambda w_{n, l}^{i} v_{2 x}^{n}\left(g^{l j} \mu+c_{s}^{l j} v_{2 x}^{s}\right)+\partial\left(w_{n, l}^{i} v_{2 x}^{n}\right)\left(g^{l j} \mu+c_{s}^{l j} v_{2 x}^{s}\right)\right) \\
=w_{m}^{k} v_{2 x}^{m} w_{n, l}^{i} v_{2 x}^{n}\left(g^{l j} \mu+c_{s}^{l j} v_{2 x}^{s}\right)+ \\
w_{m}^{k} v_{2 x}^{m}(\lambda+\mu+\partial)^{-1}\left(-\lambda w_{n, l}^{i} v_{2 x}^{n} c_{s}^{l j} v_{2 x}^{s}-\partial\left(w_{n, l}^{i} v_{2 x}^{n}\right) c_{s}^{l j} v_{2 x}^{s}\right) \\
-w_{m}^{k} v_{2 x}^{m}(\lambda+\mu+\partial)^{-1}\left(\mu \lambda w_{n, l}^{i} v_{2 x}^{n} g^{l j}+\mu \partial\left(w_{n, l}^{i} v_{2 x}^{n}\right) g^{l j}\right) .
\end{gathered}
$$

The same procedure is then repeated to eliminate $\mu$ in the last parenthesis.

We proceed similarly to compute all the terms of the nonlocal part of PVA-Jacobi identity. Finally, we get an overall expression for which we can collects the coefficients of $\lambda^{3}, \mu^{3}, \lambda^{2} \mu, \lambda \mu^{2}, \lambda^{2}, \lambda \mu, \mu^{2}, \lambda, \mu, 1,\left[(\lambda+\partial)^{-1} w_{n}^{i} v_{2 x}^{n}\right] \mu^{3},\left[(\lambda+\partial)^{-1} w_{n}^{i} v_{2 x}^{n}\right] \mu^{2},[(\lambda+$ $\left.\partial)^{-1} w_{n}^{i} v_{2 x}^{n}\right] \mu,\left[(\lambda+\partial)^{-1} w_{n}^{i} v_{2 x}^{n}\right],\left[(\mu+\partial)^{-1} w_{n}^{j} v_{2 x}^{n}\right] \lambda^{3},\left[(\mu+\partial)^{-1} w_{n}^{j} v_{2 x}^{n}\right] \lambda^{2},\left[(\mu+\partial)^{-1} w_{n}^{j} v_{2 x}^{n}\right] \lambda$, $\left[(\mu+\partial)^{-1} w_{n}^{j} v_{2 x}^{n}\right], w_{s}^{k} v_{2 x}^{s}(\lambda+\mu+\partial)^{-1} \lambda^{3}, w_{s}^{k} v_{2 x}^{s}(\lambda+\mu+\partial)^{-1} \lambda^{2}, w_{s}^{k} v_{2 x}^{s}(\lambda+\mu+\partial)^{-1} \lambda$, $w_{s}^{k} v_{2 x}^{s}(\lambda+\mu+\partial)^{-1}$. The last four terms, of course, mean that the coefficients are differential polynomials on which $(\lambda+\mu+\partial)^{-1}$ acts.

Under the assumption of skew-symmetry of the brackets provided by relations (11), some of the coefficients in the previous expansion are equivalent under the interchange of indices. We recall that the PVA-Jacobi identity is fulfilled if and only if the aforementioned terms vanish for all $(i, j, k)$; in particular, it is sufficient to consider the terms $\lambda^{-1}, \mu^{-1}$ and $(\lambda+\mu)^{-1}$ in the expansion of the nonlocal part, respectively. The independent coefficients that need to be set to 0 are hence the ones corresponding to $\lambda^{3}, \lambda^{2} \mu, \lambda^{2}, \lambda \mu, \lambda, \mu, 1$, $\lambda^{-1} \mu^{3}, \lambda^{-1} \mu^{2}, \lambda^{-1} \mu, \lambda^{-1}$. Moreover, each of these coefficients can be further expanded in the jet variables $v_{2 x}^{i}, v_{3 x}^{i}, v_{4 x}^{i}$ that appear in them, leading to a set of equations for $g^{i j}, c_{k}^{i j}$ and $w_{j}^{i}$.

In particular, we have the following:

Proposition 1. Assuming the skew-symmetry of the bracket (11), the vanishing of the following coefficients in the expansion of the PVA-Jacobi identity for the $\lambda$ bracket (9) is necessary and sufficient for the vanishing of the whole expression.

1. Coefficient of $\lambda^{3}: g^{i p} c_{p}^{k j}+g^{k p} c_{p}^{i j}$.

2. Coefficient of $\lambda^{2} \mu: g^{k p} c_{p}^{i j}+g^{i p} c_{p}^{j k}-g^{j p} c_{p}^{k i}$.

3. Coefficient of $\lambda u_{3 x}^{s}$ : after some manipulations involving the previous identities and their differential consequences we obtain $g^{k p} c_{p, s}^{i j}+c_{p}^{k j} g_{, s}^{p i}+c_{p}^{k i} c_{s}^{p j}-c_{p}^{i k} c_{s}^{p j}+g^{k p} w_{p}^{i} w_{s}^{j}$.

4. Coefficient of $\lambda^{-1} \mu^{3}:-\left(g^{j p} w_{p}^{k}+g^{k p} w_{p}^{j}\right)$. 
5. Coefficient of $\lambda^{-1} \mu^{2} v_{2 x}^{s}$ : after some manipulations involving the previous identities and their differential consequences we obtain $g^{k p} w_{p, s}^{j}+g_{, s}^{j p} w_{p}^{k}-c_{p}^{j k} w p_{s}+c_{p}^{k j} w_{s}^{p}$.

All other coefficients in the expansion are either algebraic/differential consequences of the former ones, or can be obtained from them by interchange of indices and the skewsymmetry of the bracket.

This finishes the proof of Theorem 1.

\section{Concluding Remarks}

- We have demonstrated that nonlocal operators (2) arise as Dirac reductions of local operators (1) to hyperplanes in the flat coordinates. It remains to be proved that every nonlocal operator (2) can be obtained by this construction.

- It makes sense to consider Hamiltonian operators with longer 'nonlocal tails' such as

$$
A=\partial_{x}\left(g^{i j} \partial_{x}+c_{k}^{i j} u_{x}^{k}+\sum_{\alpha=1}^{N} w_{\alpha k}^{i} u_{x}^{k} \partial_{x}^{-1} w_{\alpha l}^{j} u_{x}^{l}\right) \partial_{x},
$$

which can be viewed as Dirac reductions of local operators (1) to (special!) linear subspaces of codimension $N$. Thus, it was observed recently that the oriented associativity equations (which can be reduced to 6-component systems of hydrodynamic type) possess, in addition to a local first-order Hamiltonian structure [22], a third-order Hamiltonian operator with nonlocal tail of length $N=3$ [21].

Taking the 5th equation (4) for $w$,

$$
w_{i j, l}=c_{i j}^{s} w_{s l},
$$

and calculating its compatibility conditions $\left(w_{i j, l}\right)_{k}=\left(w_{i j, k}\right)_{l}$ we obtain a linear system for $w$ :

$$
\left(c_{i j, k}^{p}-c_{i j}^{q} c_{q k}^{p}\right) w_{p s}=\left(c_{i j, s}^{p}-c_{i j}^{q} c_{q s}^{p}\right) w_{p k},
$$

recall that the coefficients $c_{i j}^{p}$ are uniquely determined by the Monge metric $g_{i j}$. The analysis of this system for different Segre types of Monge metrics suggests that for $n=3$ the length $N$ of the nonlocal tail cannot exceed 1 . This implies that Theorem 3 of Section 3.2 gives a complete list of 3-component nonlocal operators of type (12). As an immediate corollary we obtain that already for $n=3$ not every Monge metric generates a nonlocal Hamiltonian operator of type (12) (note that for $n=2$ every Monge metric gives rise to a nonlocal operator with tail of length $N \leq 1$, see Section 3.1).

\section{Acknowledgements}

Matteo Casati was supported by the INdAM-Cofund-2012 Marie Curie fellowship 'MPoisCoho'. Maxim Pavlov was partially supported by the RFBR grant 17-01-00366. Raffaele Vitolo recognises financial support from the Loughborough University's Institute 
of Advanced Studies, LMS scheme 2 grant, INFN by IS-CSN4 Mathematical Methods of Nonlinear Physics, GNFM of Istituto Nazionale di Alta Matematica and Dipartimento di Matematica e Fisica "E. De Giorgi" of the Università del Salento. We thank CIRM (Trento) for their kind hospitality via the 'research in pairs' programme where this project was completed.

\section{References}

[1] A.V. Balandin, G.V. Potemin, On non-degenerate differential-geometric Poisson brackets of third order, Russian Mathematical Surveys 56, no. 5 (2001) 976-977.

[2] A. Barakat, A. De Sole, V.G. Kac, Poisson vertex algebras in the theory of Hamiltonian equations, Jpn. J. Math. 4, no. 2 (2009), no. 2, 141-252.

[3] A. De Sole, V.G. Kac, Non-local Poisson structures and applications to the theory of integrable systems, Jpn. J. Math. 8, no. 2 (2013) 233-347.

[4] I. V. Dolgachev, Classical algebraic geometry. A modern view, Cambridge University Press, Cambridge, 2012, 639 pp.

[5] P.W. Doyle, Differential geometric Poisson bivectors in one space variable, J. Math. Phys. 34, no. 4 (1993) 1314-1338.

[6] B.A. Dubrovin and S.P. Novikov, Poisson brackets of hydrodynamic type, Soviet Math. Dokl. 30, no. 3 (1984) 651-2654.

[7] B.A. Dubrovin, Geometry of 2D topological field theories, Lecture Notes in Mathematics, V.1620, Berlin, Springer, 120-348.

[8] E.V. Ferapontov, Nonlocal Hamiltonian operators of hydrodynamic type: differential geometry and applications, Amer. Math. Soc. Transl. (2) 170 (1995) 33-58.

[9] E.V. Ferapontov, C.A.P. Galvao, O. Mokhov, Y. Nutku, Bi-Hamiltonian structure of equations of associativity in 2-d topological field theory, Comm. Math. Phys. 186 (1997) 649-669.

[10] E.V. Ferapontov, J. Moss, Linearly degenerate PDEs and quadratic line complexes, Comm. Anal. Geom. 23, no.1 (2015) 91-127.

[11] E.V. Ferapontov, M. V. Pavlov, R.F. Vitolo, Projective-geometric aspects of homogeneous third-order Hamiltonian operators, J. Geom. Phys. 85 (2014) 16-28.

[12] E.V. Ferapontov, M.V. Pavlov, R.F. Vitolo, Towards the classification of homogeneous third-order Hamiltonian operators, IMRN no. 22 (2016) pp. 6829-6855; doi:10.1093/imrn/rnv369.

[13] Ferapontov, M.V. Pavlov, R.F. Vitolo, Systems of conservation laws with third-order Hamiltonian structures, Lett. Math. Phys. (2017), DOI: 10.1007/s11005-018-1054-3; arXiv:1703.06173. 
[14] I.M. Gelfand, I. Ja. Dorfman, Hamiltonian operators and algebraic structures associated with them, Funktsional. Anal. i Prilozhen. 13, no. 4 (1979) 13-30, 96.

[15] C.M. Jessop, A treatise on the line complex, Cambridge University Press 1903.

[16] J. Kalayci, Y. Nutku, Bi-Hamiltonian structure of a WDVV equation in 2d topological field theory, Phys. Lett. A 227 (1997), 177-182.

[17] J. Kalayci, Y. Nutku, Alternative bi-Hamiltonian structures for WDVV equations of associativity, J. Phys. A: Math. Gen. 31 (1998) 723-734.

[18] W.-X. Ma, S. Manukure, H.-C. Zheng, A counterpart of the Wadati-Konno-Ichikawa soliton hierarchy associated with $s o(3, R)$, Z. Naturforsch. 69a (2014) 411-419; http: //arxiv.org/abs/1405.1089.

[19] O.I. Mokhov, Symplectic and Poisson structures on loop spaces of smooth manifolds, and integrable systems, Russian Math. Surveys 53, no. 3 (1998) 515-622.

[20] M.V. Pavlov, R. Vitolo, On the bi-Hamiltonian Geometry of WDVV Equations, Lett. Math. Phys. 105, no. 8 (2015) 1135-1163.

[21] M.V. Pavlov, R.F. Vitolo, On the bi-Hamiltonian Geometry of Oriented WDVV Equations, to appear.

[22] M.V. Pavlov, A. Sergyeyev, Oriented associativity equations and symmetry consistent conjugate curvilinear coordinate nets, J. Geom. Phys. 85 (2014), 46-59.

[23] G. V. Potemin, On Poisson brackets of differential-geometric type, Soviet Math. Dokl. 33 (1986) 30-33.

[24] G.V. Potemin, On third-order Poisson brackets of differential geometry, Russ. Math. Surv. 52 (1997) 617-618.

[25] G.V. Potemin, Some aspects of differential geometry and algebraic geometry in the theory of solitons. PhD Thesis, Moscow, Moscow State University (1991) 99 pages.

[26] M. Wadati, K. Konno, Y. H. Ichikawa, New integrable nonlinear evolution equations, J. Phys. Soc. Japan 47, no. 5 (1979) 1698-1700. 